\title{
Quasicrystal: a beautiful morphology and diffraction pattern
}

\begin{abstract}
The synthesis of quasicrystalline phases in complex metallic alloys appears to be advantageous for fundamental research as well as practical application. Studies on quasicrystals and their applications have been research frontiers in chemistry, physics, mathematics, materials science and metallurgy in the past few decades. The remarkable progress has been made in the formation, growth and phase stability, structure and modeling, mathematics of quasiperiodic and a periodic structures, physical properties (transport, magnetic, dynamical, mechanical etc.), surfaces and over layers, applications etc. However, the structural solution of the quasicrystal is still under debate and it will provide a broad aspect for future development and guide the investigations of different aspects of quasicrystals. Therefore, a review on the quasicrystal may be attractive for material science \& engineering community.
\end{abstract}

Keywords: intermetallic, nanostructured, quasicrystal, mechanical, alloying/ milling, rapid solidification
Volume I Issue I - 2017

Yadav TP

Department of Physics, Banaras Hindu University, India

Correspondence: Yadav TP, Department of Physics, Institute of Science, Hydrogen Energy Centre, Centre of Advanced Study, Banaras Hindu University, Varanasi-22 1005, India, Tel 91-5422307307, Fax 91-542-2368468, Email yadavtp@gmail.com

Received: February 13, 2017 | Published: April 19, 2017

\section{Introduction}

The development of materials has provided researchers with more powerful instruments, methods and building blocks to create outstanding materials technology and devices. ${ }^{1}$ The discovery of new materials have provided scientists, maybe for the first time, with the possibility of exploring, modifying and constructing an artificial world as complex as nature from a fundamental level. The intermetallic materials, having very high specific strength but poor ductility, require special processing techniques to develop appropriate microstructure for adequate ductility or toughness for successful use in structural applications. ${ }^{2}$ Non-equilibrium processing of materials is often advantageous due to the possibility of producing metastable microstructure with improved properties..$^{3-5}$ Among the no equilibrium techniques developed during the past few decades to synthesize novel materials include rapid solidification from the liquid state, mechanical alloying/ milling, plasma processing, vapour deposition, ion or electron or neutron irradiation. ${ }^{6,7}$ As an important and emerging class of no equilibrium materials, such as quasicrystalline alloys have drawn wide attention in the recent years. ${ }^{8-10}$ Quasicrystalline materials are of interest because of their fundamentally new microstructure and phase dependent novel properties not manifested by identical materials with coarse microstructure. ${ }^{11}$ Improvement in terms of mechanical properties includes both increase in strength as well as ductility and fracture toughness due to a periodic structures. Aside from the peculiar structures, quasicrystals also exhibit much unexpected properties such as high hardness, low surface energy, high oxidation resistance, and low thermal conductivity, which make them attractive for technological applications. ${ }^{12,13}$ These materials can also be used as dispersions, coatings, functional devices, consolidated materials, etc. Rapid solidification of liquid metals and alloys can lead to refinement of grain size, formation of new metastable quasicrystalline phases by suppression of equilibrium solidification process. The quasicrystalline materials can be synthesized by mechanical alloying, which is a solid state powder processing technique involving repeated cold welding, mechanically activated interdiffusion, fragmentation and dynamic recrystallization of powder particles in a high energy ball mill, is an ideal processing route to develop nanoquasicrystalline materials at ambient temperature. . $^{14,15}$

\section{Quasicrystal}

Quasicrystal phases are a novel class of intermetallics compounds that exhibit conventionally forbidden rotational symmetries in their diffraction patterns, which are incompatible with translational periodicity. ${ }^{16}$ The breakthrough on rapidly solidified Al-14\% Mn alloys have created new concepts of ordered but no periodic atomic arrangements which exhibit sharp diffraction peaks with icoshahedral symmetry (m 35). For which he was awarded the Nobel Prize in Chemistry in 2011. Using transmission electron microscopy and associated high-energy electron diffraction, Shechtman made the key observation that rapidly-quenched $\mathrm{Al}_{86} \mathrm{Mn}_{14}$ alloy forms small particles in which the nature and relative orientations of the rotational axes could only be explained with icosahedral symmetry as shown in (Figure 1). Furthermore, Shechtman found that these particles did not exhibit twinning, an effect that can sometimes lead to spurious indications of icosahedral symmetry in crystalline materials, notably gold. The icosahedral point group is incompatible with translational periodicity. The sharpness of the diffraction peaks was explained until this time on the basis of the periodicity of the crystalline materials. The diffraction patterns simulated from these structures are startlingly close to those observed for the icosahederal phase and this phase was termed as a quasicrystal. The mathematics of diffraction does not necessarily require periodicity; this was discussed by Mackay before the discovery of quasicrystals. ${ }^{17}$ The Mackay extended the idea of Penrose, who had developed a scheme of filling space a periodically by a finite number of tiles. ${ }^{18}$ Mackay constructed the three dimensional analogue tiles and stressed their importance in the context of crystallography. A Penrose tiling is a non-periodic tiling generated by and a periodic lay down. The aperiodicity of prototypes implies that a shifted copy of a tiling will never match the original as shown (Figure 1). The tiling uses a pair of rhombuses with equal sides but different angles. Ordinary rhombus-shaped tiles can be used to tile the plane 
periodically; however using a pair of rhombuses as marked by 'A' and ' $\mathrm{B}$ ' exhibit both reflection and fivefold rotational symmetry.

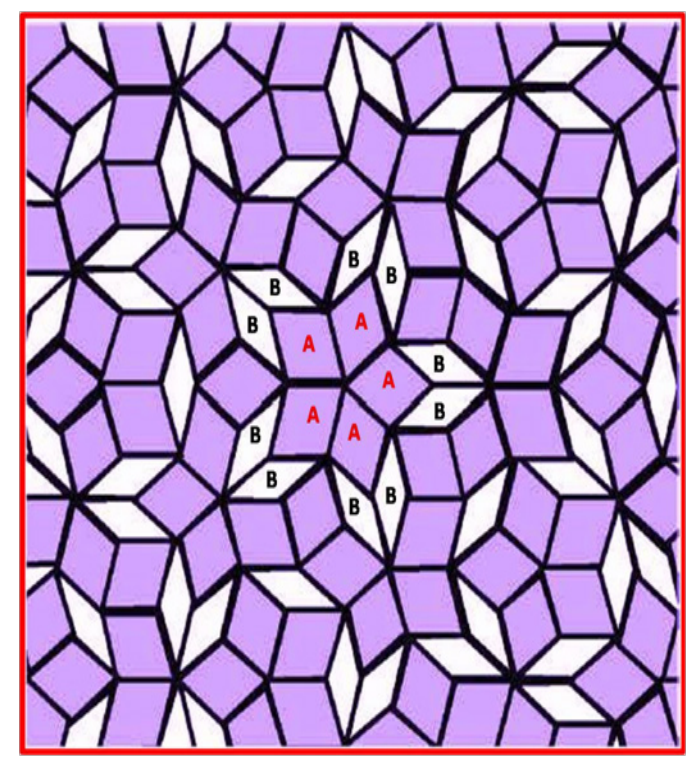

Figure I The pentagonal penrose tiling drawn uses a pair of rhombuses with equal sides but different angles with black edges.

Discovery of the first quasicrystal, it was universally accepted, though never proven, that the internal order of crystals was achieved through a periodic filling of space ${ }^{16}$ Crystallography treated order and periodicity synonymously, both serving equally to define the notion of a crystal. With that came the so-called "crystallographic restriction," stating that crystals cannot have certain forbidden symmetries, such as 5-fold rotations. The periodic nature of crystals was "confirmed" with the discovery of $\mathrm{x}$-ray crystallography and numerous other experimental techniques throughout the 20 th century. The periodic nature of materials cannot explain only on the basis of $x$-ray diffraction. The $\mathrm{x}$-ray and neutron diffraction together with Electron Microscopy observations, allowed exploring the structure of quasicrystals. The quasicrystals structure, itself be a periodic, this must avoid any lattice plane of the higher-dimensional lattice. There are several ways to mathematically define quasicrystalline patterns i.e. 'cut and project' method. Here a high-dimensional periodic function to an irrational slice and the projection points are a periodic. These functions are not exactly periodic, but they are arbitrarily close in some sense, as well as being a projection of an exactly periodic function.

Quasicrystalline materials are a novel class of intermetallics compounds that exhibit conventionally forbidden rotational symmetries in their diffraction patterns as shows in (Figure 2), which are incompatible with translational periodicity. After the discovery the first icosahedral quasicrystal, formed under rapid solidification conditions in binary $\mathrm{Al}-\mathrm{Mn}$ alloy, the thermally stable quasicrystals have been discovered in several Al based materials. ${ }^{19,20}$ A beautiful morphology of a stable $\mathrm{Al}-\mathrm{Cu}-\mathrm{Fe}$ icosahedral quasicrystal has been shown in (Figure 3) respectively. The stable quasicrystals are of great interest due to their potential for creating unusual structural forms of matter having extraordinary physical properties. The stable quasicrystalline surfaces are also important for possible technological applications. ${ }^{21}$ For example, surfaces of quasicrystals exhibit high hardness and good tribological properties such as low surface friction; they also show high oxidation resistance. ${ }^{22}$

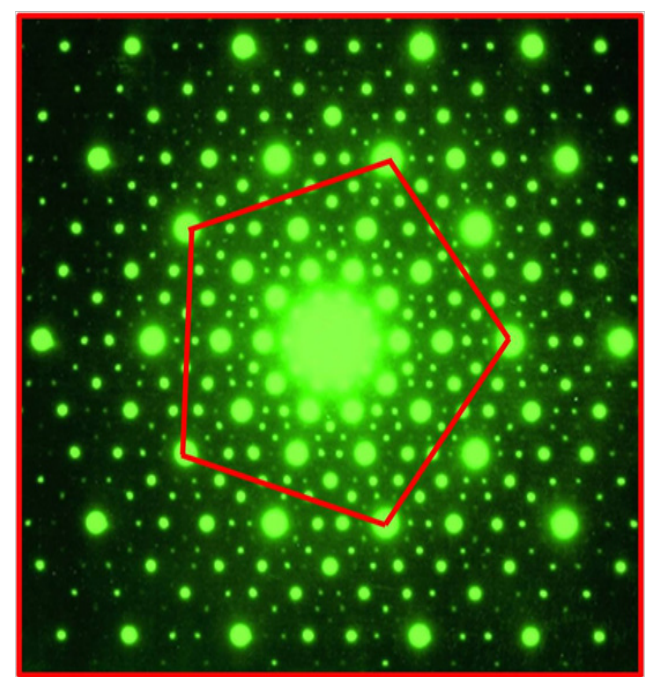

Figure $\mathbf{2}$ The transmission electron microscopy selected area electron diffraction pattern form $\mathrm{Al}-\mathrm{Cu}-\mathrm{Fe}$ icosahedral quasicrystal.

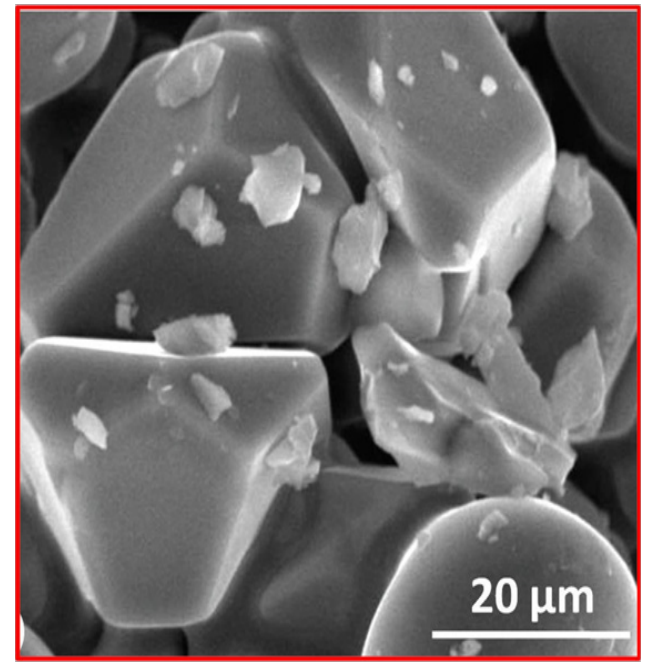

Figure 3 Surface morphology of a stable Al-Cu-Fe icosahedral quasicrystal.

\section{Potential applications of quasicrystals}

The industrial application of quasicrystals has quite limited so far, however quasicrystal exhibit interesting and sometimes unexpected properties in terms of technological applications. A few selected potential applications are as following:

\section{Hydrogen storage materials}

Quasicrystalline materials are having large free volume with quasi-periodicity and the high density of tetrahedral interstitial sites displayed by metallic alloys with quasicrystalline structure. The $\mathrm{Ti}-\mathrm{Zr}-\mathrm{Ni}$ icosahedral phase is a new, promising hydrogen storage material. ${ }^{23}$ The substitution of chemical elements in the Ti-based icosahedral phase powders may control hydrogen desorption properties. The maximum hydrogen concentration of $\mathrm{Ti}-\mathrm{Zr}-\mathrm{Ni}$ phase powders is approximately 60 at. This can be attained by either gaseous-phase or electrochemical loading of hydrogen. The incorporation of hydrogen causes the quasilattice to expand, with an increase in quasilattice constant of almost $7 \%$, from a; $=5.18$ to $5.52 \AA$, which was similar abilities for storing hydrogen in high-order crystalline approximant 
and amorphous phases of like composition. ${ }^{24}$ The hydrogen storage ability of the quasicrystal was compared to crystalline phase and found to be better than, those of the related crystalline phases in this system. They were concluded that $\mathrm{Ti}-\mathrm{Zr}-\mathrm{Ni}$ alloys may be the ideal candidates for hydrogen storage because they contain two elements, $\mathrm{Ti}$ and $\mathrm{Zr}$, which have high affinities for hydrogen. The presence of $\mathrm{Ni}$ is beneficial in that it often creates a pathway for $\mathrm{H}$ diffusion through a surface oxide layer. $\mathrm{Ti}-\mathrm{Zr}-\mathrm{Ni}$ alloys form many interesting structures, including amorphous and quasicrystalline phases and two complex crystalline phases: the $\mathrm{C} 14$ Laves phase and the $\mathrm{Ti}_{2} \mathrm{Ni}$ structure. ${ }^{25}$

\section{Coating materials}

Quasicrystals are having corrosion resistant and low coefficients of friction; therefore Quasicrystals can be applied as a surface coating for frying pans and now quasicrystal-coated frying pan appeared on the market. ${ }^{26}$ The French Company Sitram under the trademark cybernox is producing quasicrystalline coated for frying pans for the marketing. Coatings of quasicrystals, deposited by high temperature pulverization, have ideal properties for a frying pan. Surprisingly, they are also non-stick. The pseudo gap in the electronic density of states at the Fermi level in quasicrystal is also responsible for the low surface tension of the coating. The unique friction properties of decagonal quasicrystals are also useful for the coating application this is due to an intrinsic property of their peculiar crystallographic structure. The anisotropic friction properties of quasicrystals can manifest in practical situations where these materials are exposed to air environments and under loads that lead to wear of the surface films.

\section{Conclusion}

The discovery of quasicrystals has sparked debate over atomic structure, stability, and other basic scientific issues. The some fundamental about quasicrystals has yet to be settled without any ambiguity. The fact that quasicrystal structures are stable at high temperature but degenerate at low temperature lends support to the idea that they are entropic ally stabilized. However, it may still be possible to find a class of quasicrystals which are stabilized by enthalpy and hence the quasiperiodic structure would be a ground state structure.

\section{Acknowledgements}

None.

\section{Conflict of interest}

The author declares no conflict of interest.

\section{References}

1. Yadav TP, Yadav RM, Singh DP. Mechanical milling: a top down approach for the synthesis of Nanomaterials and Nanocomposites. Nanoscience and Nanotechnology. 2012;2(3):22-48

2. Yadav TP, Mukhopadhyay NK, Tiwari RS, et al. Studies on the formation and stability of nano-crystalline $\mathrm{Al}_{50} \mathrm{Cu}_{28} \mathrm{Fe}_{22}$ alloy synthesized through high-energy ball milling. Materials Science and Engineering: $A$. 2005;393(1):366-373.

3. TP Yadav, ON Srivastava. Synthesis of nanocrystalline cerium oxide by high energy ball milling. Ceramics International. 2012;38(7):5783-5789.

4. Yadav TP, Singh D, Tiwari RS, et al. Enhanced microhardness of mechanically activated carbon-quasicrystal composite. Materials Letters. 2012;80:5-8

5. Singh D, Yadav TP, Mandal RK, et al. Indentation characteristics of metallic glass and nanoquasicrystal-glass composite in $\mathrm{Zr}-\mathrm{Al}(\mathrm{Ga})-\mathrm{Cu}-\mathrm{Ni}$ alloys. Intermetallics. 2010;18(12):2445-2452.
6. Suryanarayana C. Mechanical alloying and milling. Progress in Material Science. 2001;46:1-184.

7. Loren A, Jacobson Joanna McKittrick. Rapid solidification processing. Materials Science and Engineering: R Reports. 1994;11(8):355-408.

8. Kajiwara K, Matsui Y, Yadav TP, et al. Quasicrystal as a Catalyst for the Synthesis of Carbon Nanotubes. J Nanosci Nanotechnol. 2016;16(3):3084-3089.

9. Yadav TP. Recent research and development on Quasicrystals. AIMS Materials Science. 2017;4(1):172-177.

10. Xingchen Ye, Jun Chen, Eric Irrgang M, et al. Quasicrystalline nanocrystal superlattice with partial matching rules. Nat Mater. 2017;16(2):214 219.

11. Mukhopadhyay NK, Yadav TP. Some aspects of stability and nanophase formation in quasicrystals during mechanical milling. Israel Journal of Chemistry. 2011;51(11-12):1185-1196.

12. Yadav TP, Mukhopadhyay NK, Srivastava ON. Nanocrystalline Intermetallics in $\mathrm{Al}-\mathrm{Cu}-\mathrm{Fe}-\mathrm{Cr}$ Decagonal Quasicrystalline Alloy. Materials and Manufacturing Processes. 2012;27(6):620-625.

13. Singh D, Yadav TP, Mandal RK, et al. Effect of Ti addition on the quasicrystalline phase formation and indentation characteristics of $\mathrm{Zr}_{69} \cdot{ }_{5} \mathrm{Al}_{7}$. ${ }_{5} \mathrm{Cu}_{12} \mathrm{Ni}_{11}$ alloy. Philosophical Magazine. 2011;91(19-21):2837-2845.

14. Yadav TP, Mukhopadhyay NK, Srivastava ON. Synthesis of Nano-Decagonal Quasicrystalline Material by Mechanical Alloying. Advanced Science Letters. 2014;20(5-6):1219-1223.

15. Yadav TP, Mukhopadhyay NK, Srivastava ON. Quasicrystal: Bulk to Nano. The Banaras Metallurgist. 2014;19(1):54-64.

16. Shechtman D, Bleeh I, Gratias D, et al. A Metallic Phase with LongRanged Orientational Order and Broken Translational Symmetry. Physical Review Letters. 1984;53(20):1951-1954.

17. Alan L Mackay. Crystallography and the Penrose Pattern. Physica. 1982;114:609-613.

18. Penrose Roger. The role of aesthetics in pure and applied mathematical research. Bulletin of the Institute of Mathematics and its Applications. 1974;10(2):266-271.

19. Tsai AP, Inoue A, Masumoto T. A Stable Quasicrystal in Al-Cu-Fe System. Japanese Journal of Applied Physics. 1987;26(9):L1505.

20. An-Pang Tsai, Akihisa Inoue, Tsuyoshi Masumoto. Icosahedral, Decagonal and Amorphous Phases in $\mathrm{Al}-\mathrm{Cu}-\mathrm{M}(\mathrm{M}=$ Transition Metal) Systems. Materials Transactions JIM. 1989;30(9):666-676.

21. Smerdon JA, Young KM, Lowe M, et al. Templated Quasicrystalline Molecular Ordering. Nano lett. 2014;14(3):1184-1189.

22. Jean-Marie Dubois. So Useful, Those Quasicrystals. Israel Journal of Chemistry. 2011;51(11-12):1168 - 1175.

23. Nicula R, Jianu A, Biris AR, et al. Hydrogen storage in icosahedral and related phases of rapidly solidied $\mathrm{Ti}-\mathrm{Zr}-\mathrm{Ni}$ alloys. The Eur Phys $J$ B. 1998;3(1):1-5.

24. Singh D, Shahi TP, Yadav TP, et al. Hydrogenation of $\left(\mathrm{Zr}_{69.5} \mathrm{Al}_{7 \cdot 5} \mathrm{Cu}-\right.$ $\left.{ }_{12} \mathrm{Ni}_{11}\right)_{100-\mathrm{x}} \mathrm{Ti}_{\mathrm{x}}$ quasicrystalline alloys and its effect on their structural and microhardness behavior. Journal of Non-Crystalline Solids. 2013;380:11-16.

25. Shahi RR, Yadav TP, Shaz MA, et al. Effect of processing parameter on hydrogen storage characteristics of as quenched $\mathrm{Ti}_{45} \mathrm{Zr}_{38} \mathrm{Ni}_{17}$ quasicrystalline alloys. International Journal of Hydrogen Energy. 2011;36(1):592-599.

26. Jean Marie Dubois. Properties- and applications of quasicrystals and complex metallic alloys. Chem Soc Rev. 2012;41(20):6760-6777. 